40-1| 2015

Enjeux contemporains en études irlandaises - In Memoriam Paul Brennan

\title{
«La paix sans la réconciliation » en Irlande du Nord : une faiblesse stratégique depuis 1974
}

\section{Joana Etchart}

\section{(2) OpenEdition}

Journals

Édition électronique

URL : http://journals.openedition.org/etudesirlandaises/4528

DOI : 10.4000/etudesirlandaises. 4528

ISSN : 2259-8863

Éditeur

Presses universitaires de Rennes

Édition imprimée

Date de publication : 30 juin 2015

Pagination : 209-220

ISBN : 978-2-7535-4082-8

ISSN : 0183-973X

Référence électronique

Joana Etchart, « «La paix sans la réconciliation » en Irlande du Nord : une faiblesse stratégique depuis 1974 », Études irlandaises [En ligne], 40-1 | 2015, mis en ligne le 30 juin 2017, consulté le 03 mai 2019. URL : http://journals.openedition.org/etudesirlandaises/4528; DOI : 10.4000/etudesirlandaises.4528 


\title{
"La paix sans la réconciliation ${ }^{1}$ " en Irlande du Nord : une faiblesse stratégique depuis 1974
}

\author{
Joana ETCHART \\ Université Paris-Sorbonne, Paris IV
}

\section{Résumé}

En 2014, le bilan du processus de paix nord-irlandais est mitigé. Si la paix a avancé, le deuxième objectif de la réconciliation a, semble-t-il, failli. Cet article s'interroge sur les raisons de la relative inefficacité des efforts de paix fournis depuis les années 1980 dans le champ de peacebuilding. Il propose de remonter jusqu'aux années 1969-79 pour mieux comprendre cet état de fait.

Mots clés : Irlande du Nord - post-conflit, Irlande du Nord - processus de paix, Irlande du Nord - sectarisme, Irlande du Nord - conflit

\section{Abstract}

A recent report has suggested that the peace process in Northern Ireland has focused on "peace without reconciliation". This suggests that peacebuilding activities have failed to improve community relations. How can this be, when significant programmes have been incessantly developed since the 1980s? This article offers to look back at the events that took place in the 1969-1979 period and argues that they help understand this state of affairs.

Keywords: Northern Ireland-post-conflict, Northern Ireland-peace process, Northern Ireland - sectarianism, Northern Ireland - conflict

En Irlande du Nord, malgré l'enthousiasme entourant les différents cessezle-feu (1994 et 1996) et l'accord de paix de 1998, plusieurs spécialistes reconnaissaient que le chemin de la paix serait long et compliquéz. Seize années après l'Accord du Vendredi saint, la pérennisation du gouvernement régional et le désarmement des principaux groupes paramilitaires permettent de confirmer le passage vers une société en paix. Mais des doutes persistent sur la solidité de cette paix tant les points de discorde restent nombreux entre nationalistes et unionistes. À partir de la fin des années 2000, les tensions s'accroissent autour

1. Paul Nolan, Northern Ireland Peace Monitoring Report 3, Belfast, Community Relations Council, 2014, p. 11 : "The model on offer from the top is peace without reconciliation."

2. Marianne Elliott (ed), The Long Road to Peace in Northern Ireland, Peace Lectures from the Institute of Irish Studies at Liverpool University, Liverpool, LUP, 2008. 
des points contentieux de l'organisation des défilés commémoratifs, de l'affichage de symboles identitaires ou du travail de mémoire. Les Etats britannique et irlandais peinent à faire " avancer " les représentants des partis régionaux, selon leurs propres termes ${ }^{3}$. Outre les antagonismes entre nationalistes et unionistes, d'aucuns mettent également en cause la méthode même du processus, qui a conduit à une forme de paix insatisfaisante donnant lieu à des tensions et divisions dans la société ainsi qu'entre les partis politiques ${ }^{4}$.

Pourquoi, dans le dernier rapport sur l'état de la paix publié en 2014, les auteurs regrettent-ils que le modèle proposé ne cherche à développer que « la paix sans la réconciliation ${ }^{5}$ "? Comment arrive-t-on à ce constat, alors que depuis 1995 les aides européennes $\mathrm{PEACE}^{6}$ contribuent au développement de programmes alliant les deux objectifs de paix et de réconciliation? Dans les années 2000, plusieurs critiques ont été émises à l'encontre de ces programmes, au sujet notamment de l'effet de la répartition des aides financières sur les inégalités existantes ${ }^{7}$ ou sur les tensions intercommunautaires ${ }^{8}$. L'efficacité des projets transfrontaliers a également été nuancée?. Plus récemment, des chercheurs se sont interrogés sur la place accordée aux militants issus des groupes paramilitaires ainsi qu'à l'importance donnée à leur parole dans les programmes de paix ${ }^{10}$.

Cet article souhaite apporter un éclairage nouveau sur la question des schémas d'action inadéquats et parfois inefficaces qui sont proposés dans le domaine de peacebuilding. Notre argument consiste à donner une importance primordiale aux démarches entreprises à partir de 1969 avec la création du premier organe coor-

3. Voir les propos des dirigeants des deux pays en 2013 et 2014. Par exemple : [http://www.theguardian.com/uknews/2014/oct/16/northern-ireland-talks-peace] (consulté en novembre 2014).

4. J. Ruane et J. Todd, «The Politics of Transition? Explaining political crises in the implementation of the Belfast Good Friday Agreement », Political Studies, Vol. 49, 2001, p. 923-940. Plus récemment Roy Foster, « Partnership of loss: "Ireland: The Politics of Enmity 1789-2006" by Paul Bew ", London Review of Books, 13 décembre 2007.

5. Dernier rapport sur l'état de la paix : Paul Nolan, op. cit., p. $11:$ : the model on offer from the top is peace without reconciliation."

6. L'apport global a représenté approximativement 113,7 millions d'euros par an. Michael Potter et Leigh Egerton, The EU PEACE and INTERREG Programmes in Northern Ireland, Belfast, NI Assembly Research and Information Service, 2011, p. 2.

7. Sean Byrne et Cynthia Irvin, "Economic aid and policy making: building the peace dividend in Northern Ireland ", Policy \& Politics, Vol. 29, n 4, 2001, p. 413-429.

8. Elham Atashi, « Peace dividends: the role of external aid in peacebuilding », in Maria Power (ed), Building Peace in Northern Ireland, Liverpool, LUP, 2011, p. 209-227.

9. Voir l'étude sur l'impact des aides européennes par Cathal McCall et Liam O'Dowd : « Hanging Flower Baskets, Blowing in the Wind? Third Sector Groups, Cross-Border Partnerships and the EU Peace Programmes in Ireland ", Nationalism and Ethnic Politics, vol. 14, n ${ }^{\circ}$ 1, 2008, p. 29-54.

10. Voir le débat ayant opposé les chercheurs McEvoy et Shirlow d'un côté, et Edwards et McGrattan de l'autre dans : A. Edwards et C. McGrattan, « Terroristic Narratives: On the (Re) Invention of Peace in Northern Ireland ", Terrorism and Political Violence, Vol. 23, Issue 3, 2011, p. 357-376, et K. McEvoy et P. Shirlow, "The Northern Ireland Peace Process and "Terroristic" Narratives: A Reply to Edwards and McGrattan ", Terrorism and Political Violence, Vol. 25, Issue 2, 2013, p. 161-166. 
dinateur du travail de peacebuilding, la Community Relations Commission (19691974). En 1974, le dispositif est dissous, les fonctions liées au peacebuilding fortement réduites et disséminées parmi divers services ministériels lors du passage au système de direct rule. Toutefois, l'étude de ces transformations révèle que les décisions ont été prises sans véritable réflexion cohérente et de manière décousue, par des sources d'autorité multiples. Paradoxalement, ces transformations représentent des " trajectoires $^{11}$ " importantes pour les décennies suivantes et fondent des programmes ultérieurs dans le domaine de peacebuilding.

\section{La première initiative institutionnelle de peacebuilding en Irlande du Nord : la Commission et le ministère des Relations communautaires (1969-1974)}

Entre 1969 et 1974, un dispositif ambitieux de peacebuilding est mis en place par le gouvernement régional de Stormont, à l'initiative des dirigeants britanniques $^{12}$. Il est composé d'un Ministère et d'une Commission dédiés exclusivement à la mission d'amélioration des relations communautaires. La Commission (Community Relations Commission, CRC) s'engage dans un véritable processus de réflexion et d'action sur les possibilités de faire avancer la mission de " création de relations communautaires harmonieuses ${ }^{13}$ ". Elle est extrêmement prolifique ${ }^{14}$ et ambitieuse. Sa politique principale consiste à envoyer une équipe d'animateurs dans les quartiers difficiles, les community development officers (CDOs), qui aident au développement des associations locales dans un esprit d'autogestion cher aux années $1970^{15}$. Au sein de la Commission, le président Maurice Hayes décrit leur approche en ces termes:

Il n'y a pas de solution facile ou rapide. De même qu'il n'y a pas une cause unique aux maux de notre société, il n'y aura certainement pas non

11. Notion de "trajectoire historique " utilisée par C. McGrattan, Northern Ireland 1968-2008, The Politics of Entrenchment, Basingstoke, Palgrave and Macmillan, 2010., p. 22 : "decision-making is an historical catalyst, creating causal sequences or historical "paths" by delimiting the range of options available to actors downstream and encouraging further steps in the same direction."

12. L'histoire détaillée de la création de ce dispositif a fait l'objet de recherches doctorales par l'auteur de cette étude : J. Etchart, "Les programmes de relations communautaires, community relations (CR) depuis 1969 : l'illusion d'une continuité? ", Soutenue en 2010 à l'Université Paris Sorbonne Nouvelle.

13. Community Relations Act (Northern Ireland) 1969, Chapter 23, 1(3)(a), Belfast, HMSO, 1969 : «(3) It shall be the duty of the Commission: (a) to encourage the establishment of, and assist others to take steps to secure the establishment of, harmonious community relations and to coordinate the measures adopted for that purpose by others. "

14. Les études accomplies alors par John Darby et F. W. Boal sont devenues des références. Par exemple : Community Relations Commission Research Unit, Flight, A Report on Population Movement in Belfast During August 1971, Belfast, Community Relations Commission Research Unit, 1971.

15. Maurice Hayes, " Notes for the Talk on Community Relations », Notes préparées pour une conférence, Belfast, sans éd., 14 avril 1988, p. 7. 
plus un remède unique. Le travail de la Commission n'ira pas vraiment de l'avant grâce à une seule action spectaculaire mais plutôt par une série de stratégies influant à des niveaux multiples et variés, appliquées à des échelles de temps différentes ${ }^{16}$.

La Commission s'empare d'un mode de travail social appelé community development (CD), qui prend de plus en plus d'ampleur au Royaume-Uni dans les années 1960. Selon les animateurs de la Commission nord-irlandaise, la méthode de CD permet d'aider les groupes associatifs à devenir plus conscients des différentes étapes de leur développement car elle encourage d'abord " la prise de conscience des besoins de la communauté et des individus" ainsi que "l'identification et le développement des ressources internes et externes aux communautés qui sont nécessaires pour satisfaire ces besoins ${ }^{17}$ ". Les animateurs de la Commission insistent sur la participation des communautés représentées par des associations dans les processus de prise de décision, notamment lors des étapes de la création des programmes et du financement ${ }^{18}$. En l'occurrence, il s'agit d'une véritable stratégie de peacebuilding. Par l'entremise de l'animateur de la Commission, les membres de l'association participent à une étape de définition du problème et analysent ensuite les effets des actions entamées. La discussion favorise la sélection d'une action non-violente. Généralement, les résultats du travail de la Commission sont positifs. Joe Camplisson, un animateur, déclare avoir vu " les attitudes changer de façon spectaculaire ${ }^{19}$ ". Mais la stratégie adoptée par la Commission comporte un potentiel contestataire important et dérange dès 1973.

16. Community Relations Commission, First Annual Report - 3rd December 1969 to 31st December 1970, Belfast, Northern Ireland Community Relations Commission, 1971, p. 3: "There is no easy or quick solution. As there is no one single cause for the ills of our society, there is unlikely to be any one single cure. The work of the Commission will be advanced not so much by a single dramatic act as by a whole series of strategies at many different levels enacted on various time scales."

17. Community Relations Commission, Community Development and Community Relations in Northern IrelandSome Proposals, Belfast, Northern Ireland Community Relations Commission, 1974, p. 4: "(a) The promotion of awareness of community and individual needs. (b) The identification and development of resources internal and external to communities which are needed to meet those needs. "

18. Community Relations Commission, Community Development and Community Relations in Northern Ireland - Some Proposals, op. cit., p. 4 : «(c) The growth of confidence and ability of individuals and communities to tackle community problems through self-help. (d) The development of Local Leadership, and encouragement of that leadership to work with their communities in seeking to insure that adequate resources are made available to tackle community problems and family/individual needs. (e) The promotion of awareness on the part of statutory and voluntary agencies that individuals and communities have a right to be involved in the way services are provided and to participate in the sharing of resources."

19. Joe Camplisson, "A Job for Peace. An Aspect of the C.D. Work of the N.I.C.R.C. ", non publié, 1974 : "It is at this level of development work that I have seen dramatic changes in attitudes taking place ", document cité dans F.L. Boyle, "Community Development and Community Relations. The Community Development Programme of the Northern Ireland Community Relations Commission: an Introductory Study ", PhD non publié, New University of Ulster, Coleraine, 1976, p. 31. 


\section{La dissolution de la Commission : un processus décisionnel confus}

Malgré un travail extrêmement actif, le travail de la Commission est entravé à partir de 1973 notamment par le ministère naissant. Dans le cadre du nouveau gouvernement régional mis en place en $1973^{20}$, Ivan Cooper du parti nationaliste SDLP (Social Democratic and Labour Party) est nommé ministre des relations communautaires. Or, Cooper annonce en avril 1974 la suppression de l'équipe de CDO et l'abandon du programme de CD mis en place par la Commission ${ }^{21}$. Comment expliquer cette prise de position? Selon le raisonnement du ministre Cooper, il fallait, en 1969, un organe qui soit indépendant du gouvernement unioniste afin de recueillir la confiance des catholiques. Mais, les catholiques faisant désormais partie intégrante du nouveau gouvernement en 1973, Cooper conclut que "personne n'a plus besoin de se sentir exclu du gouvernement ou de craindre que celui-ci lui soit hostile ${ }^{22}$."

Toutefois, les ambitions de Cooper sont stoppées par l'effondrement du gouvernement régional en mai $1974^{23}$. C'est le gouvernement britannique d'Harold Wilson qui dissout formellement le dispositif en décembre 1974. Certains axes de travail de la Commission dérangent, notamment lorsque les CDO cherchent à encourager le développement de groupes associatifs dans les quartiers où grandissent désormais les discours et pratiques justifiant l'action paramilitaire. Ces évolutions conduisent à marquer une rupture historique dans la prise en charge du travail associatif dans le domaine de peacebuilding. Lorsque le système adminis-

20. Des élections ont lieu en juin 1973 afin de choisir les membres de la nouvelle assemblée. Cependant, certains unionistes contestent quelques points du projet britannique, dont le partage du pouvoir dans les instances exécutives (power-sharing) et la dimension irlandaise. L'exécutif ainsi que des délégués des gouvernements de Londres et de Dublin se réunissent à Sunningdale, en Angleterre, en décembre 1973, pour discuter des modalités de collaboration entre le Nord de l'Irlande et le Sud (Council of Ireland).

21. Discours d'Ivan Cooper disponible dans Northern Ireland Assembly: Official Report, vol. 3, n 1, Belfast, Northern Ireland Assembly, 3 avril 1974.

22. Ivan Cooper, Northern Ireland Assembly Reports, 3 avril 1974, cité dans Bill Rolston, "Reforming the Orange State: Problems of the Northern Ireland Community Relations Commission ", Thames Papers in Social Analysis, Series 1: Northern Ireland, 1977, p. 69 : " there is no longer any need for any section of the community to feel excluded from government or to fear that government may be unsympathetic to them."

23. À partir de mars 1974, un nouveau groupe réunissant unionistes et loyalistes, appelé Ulster Workers' Council (UWC), menace d'organiser des actes de protestation dans la rue afin d'exprimer son rejet des principes contenus dans les accords de Sunningdale, notamment le principe du partage du pouvoir et l'instauration du Council of Ireland. La grève lancée par l'UWC débute le 15 mai 1974 dans les ports. Pendant plus de dix jours des routes sont barricadées, l'électricité coupée par intermittence et les dépôts d'essence bloqués. La grève s'étend à d'autres secteurs professionnels). On parle de paralysie de la province. La tension augmente le 17 mai lorsque plusieurs bombes explosent en République d'Irlande à Dublin et à Monaghan, tuant trente-trois personnes civiles. Cette pression conduit à la chute de l'exécutif en Irlande du Nord le 28 mai 1974, date à laquelle le système de gestion administrative de direct rule est réintroduit par le gouvernement britannique travailliste d'Harold Wilson. 
tratif de direct rule est pérennisé après 1974 , le rapport aux associations se trouve lentement formalisé vers un cadre d'administration centralisé et très contrôlé.

À cause également du contexte économique difficile, l'innovation n'est plus de mise et les activités des associations désormais financées par les ministères doivent correspondre aux missions des principaux ministères financeurs. Leur portée devient relativement limitée. La mission des relations communautaires se trouve marginalisée, en dépit du constat alarmant fait par plusieurs observateurs sur la radicalisation des tensions communautaires ${ }^{24}$.

Néanmoins, l'étude des transformations opérées dans le domaine de peacebuilding démontre que les sources d'autorité sont multiples et le processus décisionnel confus et décousu. Par exemple, l'annonce de Cooper en 1974 n'est pas suivie d'une application immédiate, ainsi la Commission continue d'exercer jusqu'à sa dissolution en décembre. Le ministère de l'Education hérite de « la responsabilité de définir et financer les programmes d'amélioration des relations communautaires $^{25}$ ", sans que cette responsabilité ne soit davantage définie. Cette réforme, opérée dans le contexte de la consolidation du système de direct rule, confirme le passage vers un mode de gestion classique et centralisé, où les programmes de peacebuilding disparaissent progressivement.

Au cours d'un entretien effectué en 2006, Hugh Frazer, directeur de la fondation Northern Ireland Voluntary Trust (NIVT) de 1979 à 1987, reconnaît que les ministres et les fonctionnaires étaient devenus craintifs : " Mais le type d'approche stratégique et politique que la Community Relations Commission avait commencé à développer ne correspondait pas à leur ambition et ils ne voulaient certainement pas de nouvelles sources de difficultés ${ }^{26}$. " L'approche audacieuse et ambitieuse proposée en 1969 n'est plus de mise. Dans le domaine de peacebuilding, la rupture opérée en 1974 représente une nouvelle " trajectoire historique » qui favorisera désormais une approche consensuelle et prudente.

24. John Darby, Geoffrey Morris, "Intimidation in Housing ", Community Forum, n 2, 1973, p. 7-11; H. A. Lyons, "Riots and Rioters in Belfast ", Community Forum, n², Belfast, Northern Ireland Community Relations Commission, 1973, p. 12-14.

25. Roland Moyle, Parliamentary Debates (Hansard), Fifth Series - vol. 888, House of Commons, Official Report, Session 1974-75, Londres, H.M.S.O., 1975, col. 978: "Article 4(2) gives the Department of Education the function of formulating and sponsoring policies for the improvement of community relations in Northern Ireland. This was the general statutory function of the Department of Community Relations. It is re-enacted here in order to make it clear that the Department of Education will pursue the same overall ends and objectives as the Department of Community Relations."

26. Hugh Frazer, Entretien (2006) : "But this sort of political strategic approach which the CRC were beginning to develop, that wasn't their ambition and they certainly didn't want anything that would create that many difficulties for them again." 


\section{La lente disparition des objectifs de peacebuilding : une prise en charge classique et centralisée des initiatives associatives dans la période 1974-1979}

Après 1974, l'aide apportée aux initiatives de peacebuilding n'est plus prise en charge par un seul dispositif identifiable mais se trouve désormais disséminée parmi les différents services des ministères de l'Éducation et de la Santé (Department of Education Northern Ireland, DENI, et Department of Health and Social Services, DHSS). Dans le cadre de ce modèle de travail essentiellement adapté aux besoins institutionnels, le rapport entre l'organisme distributeur d'aides et la population est conçu de manière peu démocratique ${ }^{27}$. L'objectif des programmes consiste désormais à obtenir l'adhésion des associations aux projets classiques tels que l'assistance aux personnes âgées. La nécessité de mener à bien ces missions n'est pas remise en question; il est même primordial qu'elles soient consolidées dans certains quartiers en Irlande du Nord. Mais, d'aucuns reprochent aux pouvoirs publics de ne pas consulter les militants sur leurs besoins, et de nier le contexte de conflit ${ }^{28}$. Généralement, les projets associatifs sont financés en fonction de critères prédéfinis par les différents services ministériels ${ }^{29}$.

Le ministère de l'Éducation, qui avait hérité de la mission d'amélioration des relations communautaires, y consacre entre 1979 et 1985 une partie de son budget constamment inférieure à $0,1 \%{ }^{30}$. Mais la transformation principale opérée après 1974 concerne la fragmentation des services impliqués dans la distribution des aides. Le schéma suivant réunit les aides attribuées au travail associatif classique, prolongeant les activités des services ministériels, ainsi que celles plus spécifiques (community development ou community relations). Le réseau de distribution des aides devient éclaté et complexe (figure 1).

En plus des ministères, les fonctionnaires des ministères et les élus locaux représentent les autres interlocuteurs possibles pour les groupes associatifs. Minis-

27. Par exemple, les personnes responsables du travail associatif au sein des boards (instance régionale dépendant du ministère de la Santé) ne sont pas élues mais choisies par le ministère de la Santé, contrairement au système en cours en Angleterre (où certains membres sont élus). Gary Craig, "Community Work and the State », Community Development Journal, vol.24, $\mathrm{n}^{\circ} 1$, janvier 1989, p. 5 et Bill Rolston, «Some Thougths on the Passing of the Northern Ireland Community Relations Commission ", New Community, vol. 5, n³ a automne 1976, p. 314.

28. Tom Lovett et Robin Percival, « Politics, Conflict and Community Action in Northern Ireland ", in Paul Curno (ed), Political Issues and Community Work, Londres, Routledge \& Kegan Paul, 1978, p. 187 : « Both politicians and civil servants viewed community development as essentially a system of self-help which could assist the statutory authorities and agencies, but should remain non-political."

29. Ce phénomène fait partie de la relation liant les associations à un système gouvernemental quel qu'il soit (nordirlandais ou autre). Le risque de se voir imposer des critères existe toujours, dans plusieurs pays. Il a souvent été critiqué. Certains perçoivent même cela comme une volonté de la part des services gouvernementaux de mieux contrôler la société civile. Cette contrainte peut avoir des effets négatifs dans un contexte de société en conflit.

30. Northern Ireland Appropriation Accounts 1979-80, Londres, HMSO, 1980 et les éditions publiées en 1981, 1982, 1983, 1984 et 1985. 
Figure 1 : Les différentes aides institutionnelles apportées au travail associatif après 1974 (Source : Schéma synthétique établi par l'auteur; Légendel NIO : Northern Ireland Office; HSS : Health and Social Services; CR : community relations; CD : community development

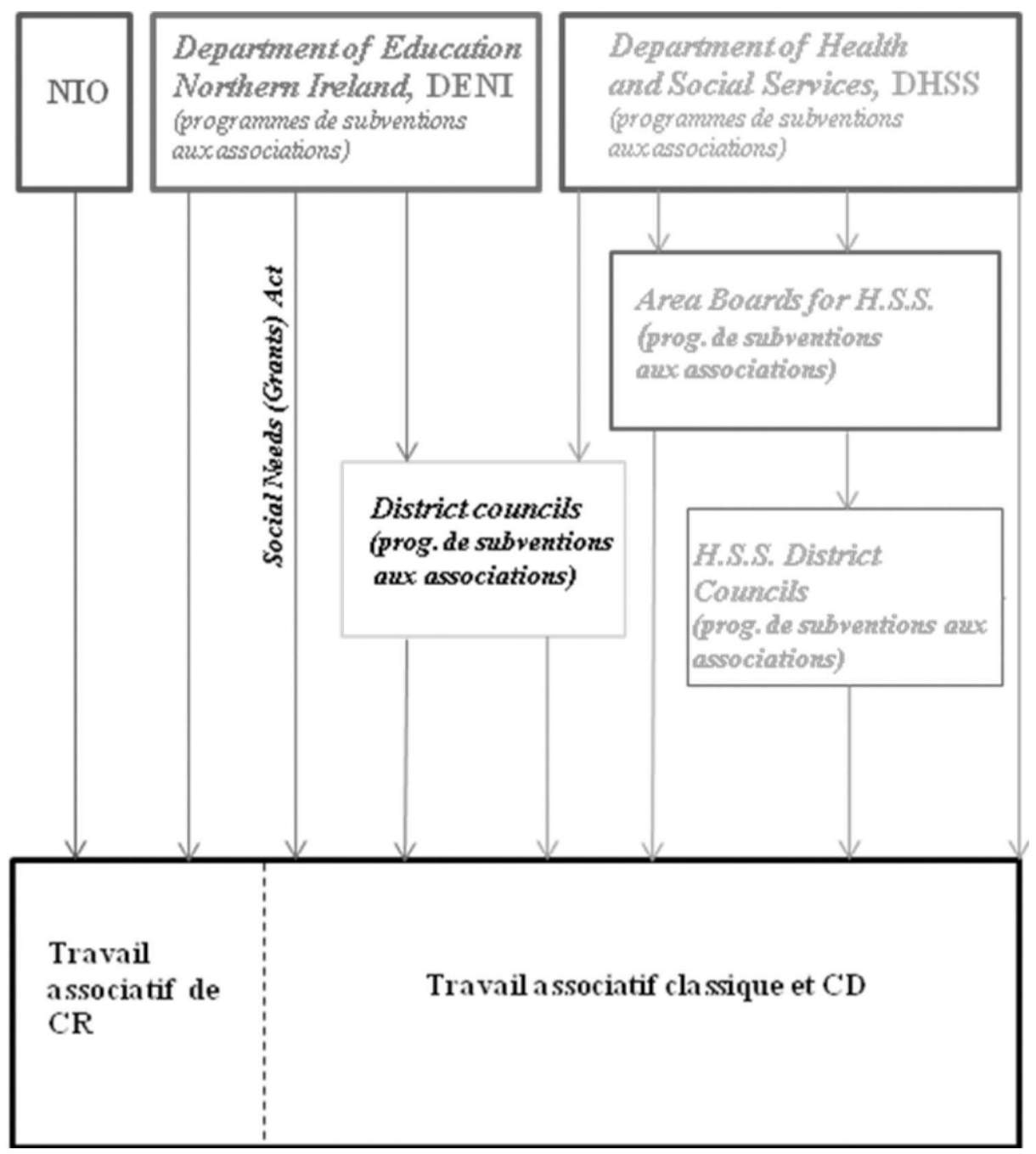

térielles, régionales ou locales, les structures d'aide varient autour d'objectifs parfois similaires. Généralement, les témoignages de l'époque exposent un système opaque et détaché des besoins du mouvement associatif ${ }^{31}$.

31. Par exemple : C. Mulrine, J. O’Neill, B. Rolston, A. Kilmurray, A Report in Funding and Support for Community Development in Northern Ireland, Belfast, Community Relations Review Group (Northern Ireland), 1991; Community Action in Northern Ireland (ed), Community Work in a Divided Society, Belfast, Farset Co-operative Press, 1981. 


\section{圈 Un modèle d'action renforcé dans les années 1980}

Les modalités de fonctionnement du système mis en place après 1974 sont perpétuées dans les années 1980 dans le cadre des aides européennes pour le développement économique de la région ${ }^{32}$. L'amélioration des relations communautaires réapparaît dans le cadre du premier plan stratégique européen Community Support Framework (1989-1993) ${ }^{33}$. Il se trouve inséré dans un projet plus vaste de développement économique ${ }^{34}$.

Mais les caractéristiques suivantes sont conservées : l'accent sur des missions classiques, une préférence pour les associations établies dont les missions sont " lisibles " par les pouvoirs publics et l'absence de soutien aux groupes associatifs alternatifs ou naissants. De plus, la fragmentation structurelle héritée de la période 1974-1979 est reproduite et renforcée lors de la redistribution des aides européennes dans la région. Par exemple, après 1985, au modèle préexistant de distribution des aides (ici en noir), s'ajoutent plusieurs nouvelles sources de financement (ici en rouge) qui redistribuent en partie les aides européennes dans la région (figure 2).

En 1994, l'aide européenne se formalise dans le cadre du second plan stratégique. La dimension de l'aide à l'apaisement du conflit y obtient une place plus importante ${ }^{35}$. Le budget augmente, ainsi que la prise en charge par des instances régionales ${ }^{36}$. Fondés dès leur origine sur le principe du développement économique, ces programmes de paix confirment l’ascendance libérale du processus de paix nord-irlandais.

32. Le Fonds Social Européen (European Social Fund, ESF) et le Fonds Européen de Développement Régional (European Regional Development Fund, ERDF). Northern Ireland Health and Social Services, Developing Voluntary Action. A Review of the Northern Ireland Council of Social Services (NICSS), Belfast, Northern Ireland Health and Social Services, 1983, p. 48.

33. Les fonds structurels destinés à l'Irlande du Nord atteignent 550 millions de livres sterling. Department of Finance and Personnel Northern Ireland, Northern Ireland Community Support Framework 1989-93, Belfast, Department of Finance and Personnel Northern Ireland (DFPNI), 1994, chapitre 3, p. 46.

34. Ibid., p. 63 : "In the case of the PSEP, new ground has been broken in terms of designing a unique and imaginative Programme targeted at helping to ease the community divisions which have been an unhappy feature of life in Northern Ireland and an obstacle to development within the region."

35. Ibid., p. 48 : "The objective of the community relations sub-programme is to increase contact between the 2 sides of Northern Ireland's divided community and to encourage greater mutual understanding about cultural diversity. "

36. LeCentral Community Relations Unit et le ministère de l'Education (DENI). Central Community Relations Unit, Project Directory - Telling the Story of Community Relations and the Role of Europe through the PSE SubProgramme (1994-99), Belfast, Central Community Relations Unit, 1999. 
Figure 2 : Structures de financement s'ajoutant à partir de 1985 au réseau existant (Source: Schéma établi par l'auteur; Légendel DFP : ministère des Finances et du Personnel; DED : ministère du Développement Economique; DENI : ministère de l'Education; DHSS : ministère de la Santé et des Services Sociaux; DOE : ministère de l'Environnement)

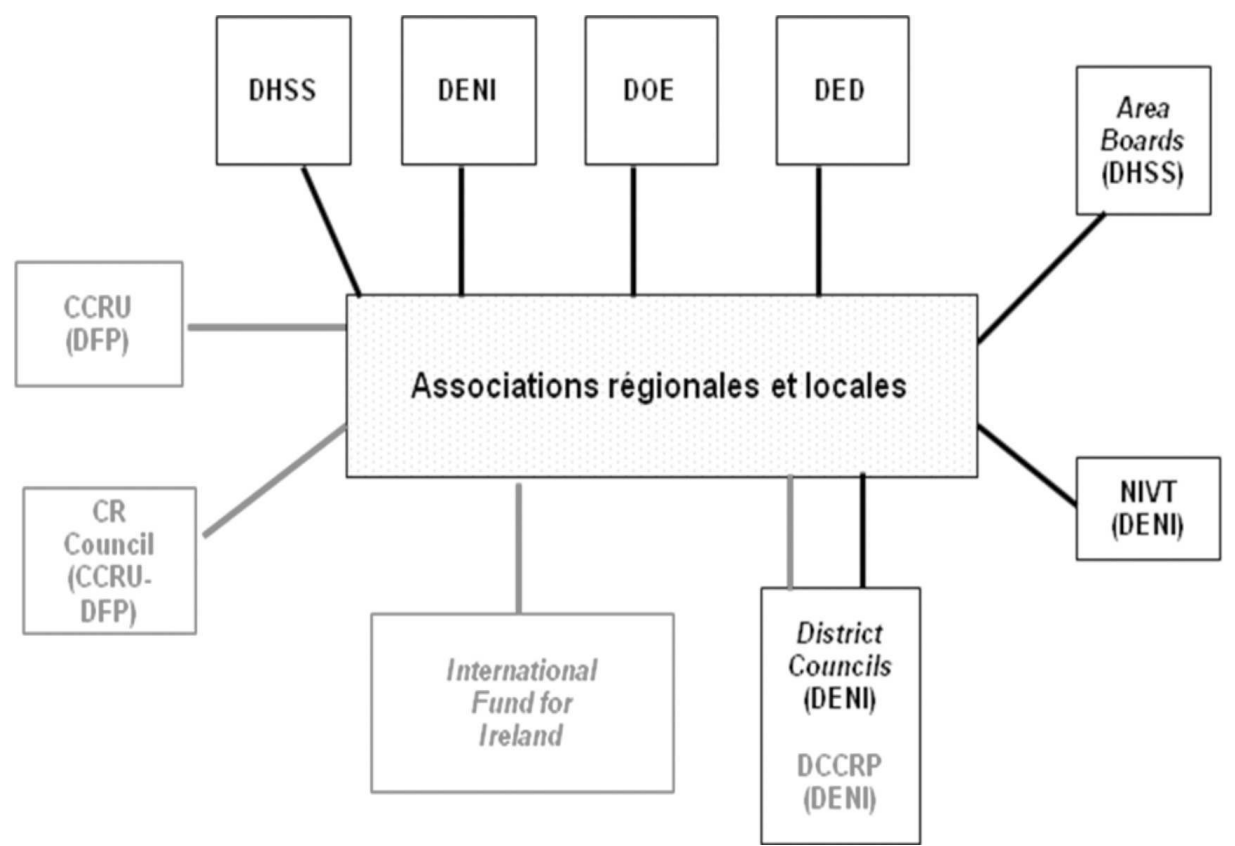

\section{La double ascendance du processus de paix : la paix libérale et les trajectoires adoptées en 1969-1979}

Audra Mitchell ${ }^{37}$ insère le processus de paix nord-irlandais dans la tradition de la paix dite " libérale ", promue principalement par les Nations Unies depuis les années $1980^{38}$. Les outils utilisés dans le cadre de cette action sont généralement la mise en place d'un cadre constitutionnel, la stabilisation de ce cadre par un système d'élections, le développement de l'économie de marché et l'accroissement des investissements.

37. Audra Mitchell, Lost in Transformation, Violent Peace and Peaceful Conflict in Northern Ireland, Basingstoke, Palgrave Macmillan, 2011.

38. E. Newman, R. Paris et O. Richmond, New Perspectives on Liberal Peacebuilding, Tokyo, United Nations University Press, 2009. Voir également Roland Paris, At War's End: Building Peace After Civil Conflict, Cambridge, Cambridge University Press, 2004 et Michael W. Doyle et Nicholas Sambani, Making War and Building Peace, Princeton, Princeton University Press, 2006; Oliver Richmond, A Post-Liberal Peace, Londres, Routledge, 2011. 
Selon Mitchell, le processus de paix nord-irlandais se définit par "la démocratisation, "les bonnes pratiques de gouvernance", le développement de l'économie et de la société civile, et, essentiellement, la sécurisation ${ }^{39}$ ". Dans le cadre des objectifs de paix fixés en 1998, la sécurisation a constitué un élément clé avec le désarmement effectif des principaux groupes armés de l'IRA en septembre 2005, de l'UVF en juin 2009 et de l'UDA en janvier 2010 ${ }^{40}$. En parallèle, en juillet 2007, l'armée britannique a transféré les compétences liées à la sécurité à la nouvelle force de police nord-irlandaise (PSNI) et mis fin à sa mission de sécurisation débutée en 1969 (Operation Banner).

Par ailleurs, les accords de 1998 définissaient le cadre d'un gouvernement régional composé d'une assemblée et d'un pouvoir exécutif. Suspendu en 2002 à cause de nombreuses impasses, il fut rétabli en 2006 grâce aux accords de Saint Andrews $^{41}$. Le 8 mai 2007, le Révérend Ian Paisley ${ }^{42}$ (Democratic Unionist Party) et Martin McGuinness (Sinn Fein) acceptèrent d'assurer conjointement la direction de l'exécutif nord-irlandais. En 2014, le gouvernement régional nord-irlandais est une institution relativement stable ${ }^{43}$.

La prédominance des objectifs libéraux de développement permet effectivement de mieux comprendre certaines limites du processus de paix nord-irlandais. Par exemple, l'insistance sur les objectifs de la sécurisation et de la stabilisation du gouvernement a conféré à ces aspects une position centrale dans le processus de paix. Ils représentent en Irlande du Nord une norme d'action, par laquelle d'autres stratégies de paix, plus localisées par exemple, peuvent subir une contrainte $^{44}$. Mitchell s'interroge également sur le lien entre le modèle normatif

39. Audra Mitchell, op. cit., p. 16 : "based on democratization, "good governance", the development of the economy and civil society, and, above all, securitization. "

40. Voir les annonces officielles des groupes paramilitaires et du groupe d'experts Independent International Commission on Decommissioning, disponibles à l'adresse : [http://cain.ulst.ac.uklevents/peaceldecommission.htm] (consulté en novembre 2014).

41. Le parlement régional (assemblée et exécutif) défini en 1998 existe de manière discontinue à partir de 2000. Finalement, l'assemblée et l'exécutif reprennent leur fonction en mai 2007 suite à un accord historique entre les partis désormais majoritaires du Sinn Fein et du Democratic Ulster Party (DUP).

42. Le dirigeant charismatique du parti DUP a néanmoins démissionné de son poste en mars 2008. Il a été remplacé par Peter Robinson.

43. Il fonctionne pour la première fois pendant un mandat entier entre 2007 et 2011. Au sein de l'Assemblée certaines décisions doivent être votées sur la base d'un accord intercommunautaire. Il s'agit du modèle de démocratie consociationnelle, théorisé par Arendt Lijphart dans les années 1960 et 1970. Voir Arendt Lijphart, «Consociational Democracy ", World Politics, 21, 1969, p. 207-225. En Irlande du Nord, quelques questions font consensus depuis 2011 lorsque les partis représentés à l'Assemblée s'accordent à réclamer davantage de pouvoir décisionnel sur les compétences fiscales. Mais ce système de fonctionnement partagé conduit parfois à des impasses et plusieurs projets de loi peuvent rester bloqués à l'état de discussions pendant des mois (voir par exemple la loi sur la réforme du gouvernement local, Local Government Act 2014, mise en route en 2002 et votée en 2014).

44. Certaines initiatives autogérées portées par des acteurs impliqués dans le conflit ont pu être marginalisées. Voir l'étude d'une initiative locale de peacebuilding parmi les populations loyalistes, J. Etchart, « Loyalism and Peacebuilding in the 2000s ", in Maria Power (ed.), Building Peace in Northern Ireland, Liverpool, Liverpool University Press, 2011, p. 131-153. 
dominant et l'émergence de discours de résistance à cette paix. En effet, quelle place est octroyée, au sein de ce modèle libéral, à la " dissidence "?

Mais les changements induits par les décisions prises dans la période charnière 1969-1979 dans le domaine de l'aide aux programmes de peacebuilding sont un facteur tout aussi important. Les trajectoires ouvertes dans les années 1970 ont conduit au développement de schémas d'intervention à portée limitée, avec des structures centralisées et fragmentées. À partir des années 1980, ces schémas ont constitué l'architecture des programmes de paix plus ambitieux. Les canaux de redistribution des aides européennes se sont accrus, mais les objectifs de chaque structure n'ont pas toujours été compris, ni mis en pratique. L'impact des programmes s'en est trouvé amoindri. Essentiellement, il n'y a pas eu de réflexion sur la rupture opérée en 1974 dans le domaine de peacebuilding, ni sur les approches écartées alors.

Ainsi, les transformations réalisées en 1969-1979 revêtent un caractère fondamental et incontournable dans l'histoire de la paix en Irlande du Nord. Si le chemin de la paix a effectivement débuté en 1969, il est encore plus long et compliqué que ce qui est généralement entendu. 